Nota

\title{
CERAS PARA CONSERVAÇÃO PÓS-COLHEITA DE FRUTOS DE ABACATEIRO CULTIVAR FUERTE, ARMAZENADOS EM TEMPERATURA AMBIENTE'
}

\author{
Marcelo Alvares de Oliveira ${ }^{2 *}$; Carlos Henrique dos Santos ${ }^{3}$; Celina Maria Henrique ${ }^{2}$ J João \\ Domingos Rodrigues ${ }^{4}$ \\ ${ }^{2}$ Pós-Graduandos do Depto. de Horticultura - FCA/UNESP, C.P. 237 - CEP: 18603-970 - Botucatu, SP. \\ ${ }^{3}$ Pós-Graduando do Depto. de Botânica - IB/UNESP - Botucatu, SP. \\ ${ }^{4}$ Depto. de Botânica - IB/UNESP - Botucatu, SP. \\ *Autor correspondente <maoliveira@laser.com.br>
}

RESUMO: Frutos de abacateiro foram colhidos dia 20/03/97. Selecionou-se 100 frutos uniformes, sendo mantidos 25 frutos para grupo controle - análise de perda de peso e 75 frutos para análises destrutivas textura, sólidos solúveis totais e $\mathrm{pH}$ realizadas a cada 4 dias. Os frutos receberam os tratamentos, com objetivo de aumentar a vida pós-colheita: Cera "Fruit Wax" 1:1 (FW1) e 1:3 (FW2); "Sparcitrus" 1 (SP1) e 1:1 (SP2); Testemunha. Até o $4^{\circ}$ dia de armazenamento não houve diferença significativa entre os valores de perda de peso dos tratamentos, sendo que ao final do armazenamento $\left(12^{\circ}\right.$ dia) o tratamento SP1 foi superior aos demais, acarretando menor perda de peso dos frutos. Não houve diferença significativa entre os valores de textura analisados dentro de cada dia de armazenamento, mas os valores decresceram durante 0 armazenamento. Para sólidos solúveis totais não observou-se aumento significativos dos teores durante 0 armazenamento, sendo que em relação aos tratamentos, apenas no $12^{\circ}$ dia para o tratamento FW2 obtiveramse valores mais elevados do que o SP1. Para o pH não houve diferença significativa entre os valores dentro dos dias de armazenamento, entretanto do ponto de colheita para $\circ 4^{\circ}$ dia de armazenamento, houve diminuição significativa, com exceção do tratamento SP1.

Palavras-chave: Persea americana, armazenamento, preservação, fisiologia, abacate

\section{WAXES FOR ENHANCED SHELFLIFE OF AVOCADO CULTIVAR FUERTE, STORED AT ROOM TEMPERATURE}

\begin{abstract}
Avocado contains fat-soluble vitamins that in general other fruits do not contain. Avocado fruits were picked and 100 uniform specimens were selected, of which 25 were used as a control group to evaluate weight loss, and 75 were used for destructive analysis. Every 4 days 25 fruits were evaluated for texture, total soluble solids and $\mathrm{pH}$. Five treatments were used to protect fruits: control; "Fruit wax"/wax 1:1 (FW1) and "Fruit wax"/wax 1:3 (FW2); "Sparcitrus/wax 1:1 (SP1) and "Sparcitrus/wax 1:3 (SP2). At the fourth storage day there was no statistical difference among the values of weight loss, however at the twelfth day, treatment SP1 was the best, with a smaller weight loss. There was no statistical difference among texture values as well as for storage day, the values however decrease during storage. Total soluble solids did not increase with storage time, until the twelfth storage day, when FR2 values were higher than those of SP1. There was no statistical difference among $\mathrm{pH}$ values for storage days, however for all treatments after the fourth storage day, the values decreased, with the exception of the SP1 treatment.

Key words: Persea americana, preservation, fisiology, fruit
\end{abstract}

\section{INTRODUÇÃO}

O abacateiro é cultivado em quase todas as regiões tropicais e subtropicais, particularmente no México, América Central, partes da América do Sul, nas Índias Ocidentais, África do Sul, Israel e no Havaí; em menor extensão, na Índia, República Malgache, Reunião, Madeira, Samoa, Taiti, Algéria, Austrália, EUA (Medina et al., 1978).

Em muitos países das Antilhas e do Oriente, o abacate é ingerido sob a forma de salada com cebola e queijo ou de sopa, ou com sal e pimenta do reino, ou mesmo em conserva. Entre nós, é mais apreciada como fruta madura adicionada de açúcar, mel ou licores.

As qualidades organolépticas, o valor nutritivo e a riqueza em vitaminas do abacate justificam plenamente a expansão do seu consumo.

O abacate contem as vitaminas lipossolúveis que em geral faltam nas outras frutas. É muito rico em vitaminas $A$ e $B$, medianamente rico em vitaminas $D$ e $E$ e muito pobre em vitamina C (Haendler, 1965 apud Medina et al 1978). O valor calórico por 100 gramas de fruto pode variar de 55 a 200 calorias (Maranca, 1978). 
O abacate é classificado como um fruto climatérico, e a textura está estreitamente relacionada com a solubilização de substâncias pécticas. Durante a maturação há a conversão da pectina insolúvel em pectina solúvel, amolecendo e diminuindo a resistência dos frutos (Chitarra \& Chitarra, 1994).

No Brasil, muito se perde da produção agrícola durante a fase pós-colheita, em função do desconhecimento de técnicas de conservação. Para a diminuição das perdas utilizam-se algumas técnicas pós-colheita, entre as quais 0 tratamento com fungicidas, controle de temperatura e umidade, aplicação de ceras, etc (Oliveira, 1996).

A aplicação de ceras é um método que começou a ser estudado na década de 80 . Apesar de se mostrar eficiente, tem como principal limitação seu custo e o possível efeito residual nos frutos. As ceras aumentam o período de conservação de frutos e hortaliças, através da diminuição da taxa transpiratória e da atividade metabólica (Oliveira, 1996).

Gayet et al. (1995) relataram que o abacate requer no máximo 10 dias a partir da colheita para atingir seu total amadurecimento, quando mantido à temperatura ambiente. Entretanto, quando os frutos são encerados, pode-se estender a vida de prateleira por um tempo maior, a qual irá depender da concentração da emulsão aplicada. Se a concentração for aumentada de 20 para $25 \%$ de cera, a vida de prateleira pode ser ampliada de 10 para 17 dias.

Joyce et al. (1995) relataram que abacates da cultivar 'Hass' armazenados à temperatura constante de $22{ }^{\circ} \mathrm{C}$ apresentaram uma perda de peso diária de $0,99 \%$, sendo que quando os mesmos foram tratados com a cera produzida pela firma Colin Campbell Chemistry, a perda de peso diária foi de $0.51 \%$.

O abacate da cultivar 'Fuerte' tem ultimamente ganhado um grande mercado nacional e mundial, devido poder ser utilizado tanto para salada, quanto para doce, além de ter um tamanho menor, que é a tendência para a diminuição de perda pós-colheita de frutas, já que pode ser consumido em uma única vez por uma única pessoa.

Com o intuito de estabelecer as condições necessárias para prolongar a conservação pós-colheita do abacate da cultivar 'Fuerte', e armazenando-os à temperatura ambiente, coduziu-se o presente trabalho.

\section{MATERIAL E MÉTODOS}

Os frutos procedentes da Fazenda Jaguacy, localizada no município de Bauru/SP, foram colhidos manualmente em 20/03/97, ao atingirem o ponto de maturação fisiológica ("de vez"), e transportados no mesmo dia para Botucatu, em caixas plásticas, para 0 Departamento de Horticultura da Faculdade de Ciências Agronômicas Campus de Botucatu - UNESP onde foi realizado o experimento. O pomar comercial possui cerca de 40.000 pés de abacate. O clima é tropical úmido e não se faz o uso de irrigação. O cultivar utilizado foi o 'Fuerte'. Após a colheita, foi feita a seleção de 100 frutos quanto a uniformidade, coloração e ponto de maturação visando a obtenção de um lote homogêneo. Para cada tratamento foram mantidos 5 frutos para acompanhamento do grupo controle e 15 frutos reservados para análises destrutivas (3 para cada tratamento -3 repetições), constituindo o grupo destrutivo. As análises foram realizadas de 4 em 4 dias.

As ceras avaliadas foram a "Fruit wax", cera utilizada para tratamento pós-colheita, classificada como cera água (solúvel em água) sendo obtida a partir da palma brasileira carnaúba e a "Sparcitrus" cera utilizada para tratamento pós-colheita, de origem não revelada.

Preliminarmente, todos os frutos foram mergulhados em água contendo fungicida de nome químico Prochloraz produzido pela Schering e de classe toxicológica I, na concentração de $0,2 \mathrm{~g} \mathrm{~L}^{-1}$ de produto comercial, durante 1 minuto, como procedimento para prevenir o aparecimento de podridões causadas por fungos. Posteriormente os frutos foram divididos em 5 tratamentos:

1)Testemunha:

2)Cera "Fruit Wax" (1:1):

3)Cera "Fruit Wax" (1:3).

4)Cera "Sparcitrus" (1):

5)Cera "Sparcitrus" (1:1).

No tratamento testemunha os frutos foram colocados diretamente em bandejas plásticas para acompanhamento do tratamento.

Para o preparo das diluições de cera "Fruit Wax" (Tratamentos 2 e 3 ) utilizou-se água a temperatura ambiente. Para obter a concentração da cera de (1:1) diluiu-se uma parte da cera para uma parte de água. Para a concentração 1:3 diluiu-se uma parte de cera para três partes de água (Spartan do Brasil - Catalogo técnico com referências para as diluições da cera "Fruit Wax" utilizadas para algumas frutas). Depois da pulverização dos frutos com as soluções de cera, os mesmos foram colocados em bancada para secar a temperatura ambiente. Em seguida os frutos foram colocados em bandejas plásticas para acompanhamento dos tratamentos.

No tratamento com a cera "Sparcitrus" (1) utilizouse a cera sem diluição. Para a concentração 1:1 diluiu-se uma parte de cera para uma parte de água (Spartan do Brasil Catalogo técnico com referências para as diluições da cera "Sparcitrus" utilizadas para algumas frutas). Depois da pulverização dos frutos com as soluções de cera, os mesmos foram colocados em bancada para secar a temperatura ambiente. Em seguida os frutos foram colocados em bandejas plásticas para acompanhamento dos tratamentos.

Em frutos de todos os tratamentos foram feitas determinações de 4 em 4 dias de perda de peso (grupo controle); e de textura (texturômetro Stevens), sólidos solúveis totais (Association of Official Analytical Chemistry, 1970), índice pH (Instituto Adolfo Lutz, 1985) - (grupo destrutivo).

No período de 12 dias relativos à realização do experimento foram registradas temperaturas ambientais que variaram de $25^{\circ} \mathrm{C} \pm 4^{\circ} \mathrm{C}$ e valores de umidade relativa que variaram de $64,5 \% \pm 11,5 \%$, correspondendo aos meses de março/abril. 
O delineamento estatístico utilizado foi inteiramente casualizado, seguido do teste de Tukey a $5 \%$. Para os dados de análise de perda de peso, utilizou-se regressão polinomial para traçar a curva de perda de peso de cada tratamento (Nogueira \& Piedade, 1992).

\section{RESULTADOS E DISCUSSÃO}

Desde o início do armazenamento, verificou-se em todos os tratamentos com as ceras menores valores de perda de peso dos frutos do que no tratamento testemunha (1,31\% ao dia). (Figura 1$)$. No $12^{\circ}$ dia de armazenamento os frutos do tratamento "Sparcitrus 1" apresentaram menores valores de perda de peso $(0,43 \%$ ao dia), seguidos dos frutos dos tratamentos "Fruit wax 1 " $(0,56 \%$ ao dia) e "Sparcitrus 2" (0,60\% ao dia) (Figura 1). Estes dados são concordantes com os de Gayet et al. (1995) que afirmaram que a utilização de cera aumenta a vida de prateleira dos abacates, já que com a utilização da mesma se observa menores perdas de peso. Estes dados também são concordantes com Joyce et al. (1995) que relataram que abacates da cultivar 'Hass' que foram tratados com a cera, obtiveram perda de peso diária de $0.51 \%$, bem menor a perda de peso dos frutos não tratados que atingiram a taxa diária de $0,99 \%$. As curvas de perda de peso dos frutos tratados com as cera, foram menos acentuadas do que a curva do tratamento testemunha, caracterizando a menor perda de peso dos frutos que foram tratados com as ceras. O tratamento "Sparcitrus 1" foi o mais eficiente em relação a diminuição da perda de peso dos frutos, o que ficou bem caracterizado através da menor inclinação da curva em relação aos demais tratamentos (Figura 1).

Com relação aos teores de sólidos solúveis totais, não se observou aumento significativo dos teores nos frutos durante os dias de armazenamento (TABELA 1).

Até $08^{0}$ dia de armazenamento não houve diferença significativa entre os teores médios de sólidos solúveis totais dos frutos, sendo que ao $12^{0}$ dia de armazenamento os frutos dos tratamentos testemunha e "Fruit wax 2" tinham teores mais elevados do que o do "Sparcitrus 1" (TABELA 1). Isto mostra que os frutos do

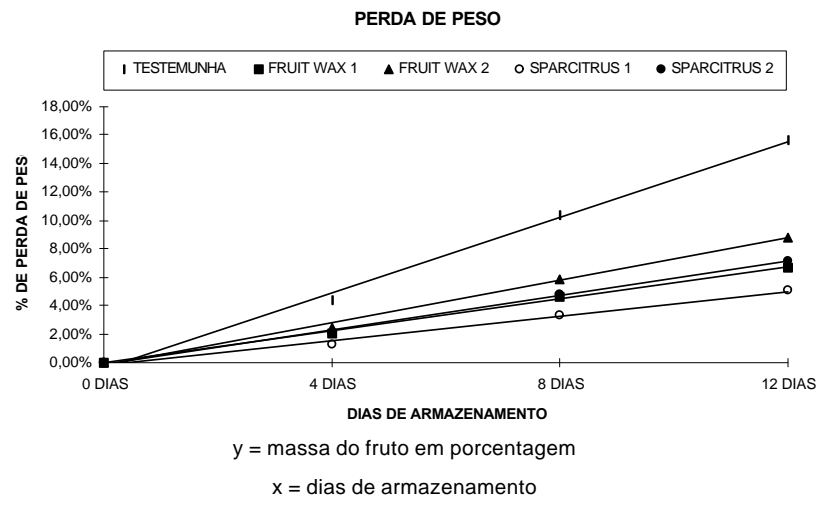

Figura 1 - Curvas de perdas de peso dos frutos relativas aos tratamentos e suas respectivas equações de regressão. tratamento "Sparcitrus 1" tiveram um retardo no amadurecimento, atingindo no $12^{\circ}$ dia de armazenamento teores ainda menores de sólidos solúveis totais do que os frutos dos tratamentos testemunha e "Fruit wax 2". Os frutos dos tratamentos "Fruit wax 1" e "Sparcitrus 2" atingiram o final do armazenamento sem diferir estatisticamente de nenhum dos tratamentos em relação a este atributo.

Com relação aos índices pH dos frutos, não se observou diferença significativa entre os valores dentro dos dias de armazenamento (TABELA 2).

Entretanto em relação aos tratamentos, observou-se que do ponto de colheita para o $4^{0}$ dia de armazenamento houve diminuição significativa entre os índices de $\mathrm{pH}$ dos frutos, com exceção do tratamento "Sparcitrus 1". Com a maturação esperava-se um aumento nos valores de $\mathrm{pH}$, conseqüência da diminuição de acidez total titulável que ocorre normalmente em todos os frutos durante o amadurecimento, entretanto isto só ficou bem caracterizado para todos os tratamentos quando se comparou o $4^{\circ}$ dia de armazenamento para os conseqüentes, $8^{0}$ e $12^{\circ}$.

Em relação aos valores de textura observados nos frutos dentro dos dias de armazenamento, não houve diferença significativa (TABELA 3).

TABELA 1 - Valores médios de sólidos solúveis totais ('Brix) dos frutos de abacate 'Fuerte' nos tratamentos.

\begin{tabular}{lcccc}
\hline TRATAMENTOS & \multicolumn{4}{c}{ DIAS DE ARMAZENAMENTO } \\
\cline { 2 - 4 } & 0 & 4 & 8 & 12 \\
\hline TESTEMUNHA & 6,68 aA & 6,73 aA & 7,75 aA & 7,08 aA \\
"FRUIT WAX 1" & 6,68 aA & 6,84 aA & 6,95 aA & 6,33 abA \\
"FRUIT WAX 2" & 6,68 aA & 6,63 aA & 7,58 aA 7,58 aA \\
"SPARCITRUS 1" & 6,68 aA & 7,08 aA & 6,87 aA & 6,00 bA \\
"SPARCITRUS 2" & 6,68 aA & 7,16 aA & 7,70 aA & 7,04 abA \\
\hline
\end{tabular}

- médias seguidas das mesmas letras não diferem significativamente a $5 \%$ pelo teste de Tukey

- letra minúscula é comparação na coluna

- letra maiúscula é comparação na linha

- $\mathrm{CV}=8,43 \%$

TABELA 2 - Valores médios de pH dos frutos de abacate 'Fuerte' nos tratamentos.

\begin{tabular}{llccc}
\hline TRATAMENTOS & \multicolumn{4}{c}{ DIAS DE ARMAZENAMENTO } \\
\cline { 2 - 5 } & 0 & 4 & 8 & 12 \\
\hline TESTEMUNHA & 6,47 aA & 5,73 aB & 6,17 aAB & 6,17 aAB \\
"FRUIT WAX 1" & 6,47 aA & 5,83 aB & 6,12 aAB & 6,33 aA \\
"FRUIT WAX 2" & 6,47 aA & 5,73 aB & 6,04 aAB & 6,17 aAB \\
"SPARCITRUS 1" & 6,47 aA & 6,04 aA & 6,03 aA & 6,32 aA \\
"SPARCITRUS 2" & 6,47 aA & 5,81 aB & 5,82 aB & 6,23 aAB \\
\hline - médias seguidas das mesmas letras não diferem significativamente \\
a 5\% pelo teste de Tukey \\
- letra minúscula é comparação na coluna \\
- letra maiúscula é comparação na linha \\
- CV = 3,43\%
\end{tabular}


TABELA 3 - Valores médios de textura (grama força-1) dos frutos de abacate 'Fuerte' nos tratamentos.

\begin{tabular}{lcccc}
\hline TRATAMENTOS & \multicolumn{4}{c}{ DIAS DE ARMAZENAMENTO } \\
\cline { 2 - 5 } & 0 & 4 & 8 & 12 \\
\hline TESTEMUNHA & $877,33 \mathrm{aA}$ & $692,67 \mathrm{aAB}$ & $343,00 \mathrm{aBC}$ & $221,00 \mathrm{aC}$ \\
"FRUIT WAX 1" & $877,33 \mathrm{aA}$ & $787,33 \mathrm{aA}$ & $418,00 \mathrm{aA}$ & $461,67 \mathrm{aA}$ \\
"FRUIT WAX 2" & $877,33 \mathrm{aA}$ & $797,00 \mathrm{aA}$ & $533,33 \mathrm{aA}$ & $59,33 \mathrm{aB}$ \\
"SPARCITRUS 1" & $877,33 \mathrm{aA}$ & $846,33 \mathrm{aA}$ & $757,67 \mathrm{aA}$ & $507,67 \mathrm{aA}$ \\
"SPARCITRUS 2" & $877,33 \mathrm{aA}$ & $842,00 \mathrm{aA}$ & $547,67 \mathrm{aA}$ & $68,67 \mathrm{aB}$ \\
\hline
\end{tabular}

- médias seguidas das mesmas letras não diferem significativamente a $5 \%$ pelo teste de Tukey

- letra minúscula é comparação na coluna

- letra maiúscula é comparação na linha

- $\mathrm{CV}=35,37 \%$

Entretanto, em relação aos dias de armazenamento, observou-se que os valores decresceram, embora diferenças significativas tenham sido observadas apenas para os frutos dos tratamentos testemunha, "Fruit wax 2" e "Sparcitrus 2". Os dados refletem o que Chitarra \& Chitarra (1994) afirmaram, que a textura está estreitamente relacionada com a solubilização de substâncias pécticas e que durante a maturação ocorre a conversão da pectina insolúvel em pectina solúvel, amolecendo e diminuindo a resistência dos frutos.

\section{CONCLUSÃO}

A utilização das ceras "Fruit wax" e "Sparcitrus" foi eficiente na redução da perda de peso dos frutos, sem interferir nos teores de sólidos solúveis totais, índice $\mathrm{pH}$ e textura. A utilização da "Sparcitrus" na concentração mais elevada, além de mais eficiente na redução de perda de peso dos frutos, proporcionou um maior brilho aos mesmos.

\section{REFERÊNCIAS BIBLIOGRÁFICAS}

ASSOCIATION OF OFFICIAL ANALYTICAL CHEMISTRY. Official methods as analysis of the Association of Official Analytical Chemistry. 11.ed. Washington; AOAC, 1970. $1015 p$.

CHITARRA, M.I.F.; CHITARRA, A.D. Pós-colheita de frutas e hortaliças: fisiologia e manuseio. Lavras: FAEPE, 1994. 293p.
GAYET, J.P.; BLEINROTH, E.W.; MATALLO, M.; GARCIA, E.E.C.; GARCIA, A.E.; ARDITO, E.F.G.; BORDIN, M.R. Abacate para exportação: procedimentos de colheita e pós-colheita. Brasília: FRUPEX, 1995. 37p.

INSTITUTO ADOLFO LUTZ. Normas analíticas: métodos químicos e físico-químicos para análise de alimentos. 3.ed. São Paulo, 1985. v.1, 533p.

JOYCE, D.C.; SHORTER, A.J.; JONES, P.N. Effect of delayed film wrapping and waxing on the shelf life of avocado fruit. Australian Journal of Experimental Agriculture, v.35, p.657-659, 1995.

MARANCA, G. Fruticultura comercial: manga e abacate. 3.ed. São Paulo: Nobel, 1978.

MEDINA, J.C.; BLEINROTH, E.W.; TANGO, J.S.; CANTO, W.L. Abacate: da cultura ao processamento e comercialização. Campinas: ITAL, 1978. 212p.

NOGUEIRA, M.C.S.; PIEDADE, S.M.S. Curso de estatística experimental aplicada à experimentação agronômica: sequência de telas do "Sanest". Piracicaba, 1992. 121p.

OLIVEIRA, M. A. de. Utilização de películas de fécula de mandioca como alternativa à cera comercial na conservação pós-colheita de frutos de goiaba (Psidium guayava) variedade Kumagai. Piracicaba, 1996. 73p. Dissertação (Mestrado) Escola Superior de Agricultura "Luiz de Queiróz", Universidade de São Paulo.

$\overline{\text { Recebido em } 11.01 .00}$ 